in emotion processing and in inhibitory executive functions. If these disturbances are trait like in nature, consistent with endophenotype status, we might expect them to be present in subclinical depression. Following a dimensional view of depression, our objective was to identify emotion perception and cognitive markers of subclinical depression while controlling for the effects of age and gender.

Method: Subjects from the Brain Resource International Database (BRID) were tested on the standardized BRID protocols, which included assessment for level of depressed mood and performance on the computerized tests of social (facial emotion recognition) and general cognition.

Results: Regression analyses showed that higher subclinical depression was significantly predicted by both social cognition (lower accuracy for recognizing fearful expressions, as well as slower reaction time for recognizing expressions of happiness and anger) and general cognition (reduced inhibition on the go-no-go test). These findings were observed over and above the effects of age and gender. When considered together, the combination of poor fear recognition and poor inhibition made the greatest contribution to level of depression.

Conclusions: Our results provide support for an integrated model of depression, in which difficulties in both emotion processing and inhibition are defining features. Dysregulation of frontolimbic circuits may contribute to disturbed processing of salient signals of emotion and the inability to inhibit automatic responses.

$11-03$

\section{Identifying cognitive and affective markers within an integrative neuroscience model of ADHD}

\section{DF Hermens ${ }^{1,2}$, MR Kohn ${ }^{1,3,4}$, SD Clarke ${ }^{1,3,4}$, CR Clark ${ }^{5}$, E Gordon ${ }^{1,2}$, LM Williams ${ }^{1,2}$}

'The Brain Dynamics Centre, Westmead Millennium Institute, Westmead Hospita and Western Clinical School, University of Sydney, Australia; 'Brain Resource International Database, Brain Resource Company; ${ }^{3}$ Adolescent Medicine, CRASH, Westmead Hospital, Westmead, Australia; ${ }^{4}$ Children's Hospital at Westmead, Westmead, Australia; and ${ }^{5}$ Cognitive Neuroscience Unit, Flinders University, Adelaide, Australia

Background: Attention deficit hyperactivity disorder (ADHD) is a common neurodevelopmental disorder, often allied with conduct and emotional problems. Our integrative model of ADHD explores fronto-limbicstriatal networks and arousal regulation to understand poor inhibitory function in ADHD. This model pro- vides the basis to develop new and objective assessment tools that complement subjective clinical scales in diagnostic and treatment decisions. Our objective was to identify the combination of cognitive and brain function markers that distinguish ADHD. We also evaluated these markers in response to stimulants treatment.

Methods: About 175 patients with ADHD (6-16 years) tested before and after stimulant treatment were compared with 175 matched controls using the standardized Brain Resource protocols. Testing included batteries of cognitive tests and psychophysiological measures of brain function [EEG, event-related potentials (ERPs)] in response to cognitive- and emotion-related tasks.

Results: Multivariate analyses showed a profile of cognitive and brain function markers that distinguished ADHD ( $90 \%$ sensitivity, $71 \%$ specificity, 0.76 positive predictive power, 0.88 negative predictive power). This profile comprised errors of impulsivity and intrusion, with raised slow-wave EEG, altered ERPs during inhibition and emotion processing, and autonomic dysregulation. While cognitive, EEG and inhibition-related ERP markers 'normalized' following stimulants, the emotion ERP marker did not. Autonomic markers distinguished subgroups of treatment responders.

Conclusions: These findings support an integrative model of ADHD, in which impulsivity and poor inhibition are associated with dysregulation of brain function and arousal. The markers provide a platform for objective assessment to support diagnostic and treatment decisions. This work is being extended to examine whether these markers capture comorbid features of ADHD and differential response to nonstimulants.

$11-04$

\section{Identifying cognitive and affective markers within an integrative neuroscience model of Alzheimer's dementia}

\section{B Liddell ${ }^{1,2}$, J Moyle ${ }^{3}$, L Williams ${ }^{2,3}$, E Gordon'}

${ }^{1}$ The Brain Resource International Database, Brain Resource Company; ${ }^{2}$ The Brain Dynamics Centre, Westmead Millennium Institute, Westmead Hospital and Western Clinical School, University of Sydney, Australia; and

${ }^{3}$ Psychological Medicine, University of Sydney, Sydney, Australia

Objective: Alzheimer's disease (AD) is a neurodegenerative dementia subtype characterized by widespread and continuing cognitive deficits encompassing abilities in memory, language and executive function that exceed the decline observed 
in normal aging. Such decline has significant consequences for the social functioning of the individual. The objective of this study was to identify the cognitive markers that best differentiate AD from healthy controls and to determine whether affective deficits make an important additional combination to definition of AD.

Method: Nineteen patients with probable AD and 38 matched healthy control subjects were tested as part of the Brain Resource International Database. Testing included a detailed medical history, a battery of cognitive tests, for which sound psychometric properties have been established, and psychophysiological recording using EEG and event-related potential (ERP) measures in response to cognitive- and emotion-related tasks.

Results: Between-subjects ANOVAs showed that the $\mathrm{AD}$ group showed marked and pervasive deficits relative to controls, on tests spanning verbal and visual memory, working memory capacity, attention function and vigilance. In addition, the relationship between cognitive decline and general mood (depression) was also examined. Significantly, measures of working and verbal memory abilities showed an inverse relationship with markers of depression and general mood at the time of testing, whereas the opposite or no relationship between such measures was observed in the control group. These findings will be discussed in relation to the psychophysiological measures of brain function during emotion perception and working memory tasks.

Conclusions: These preliminary findings provide support for the added benefit of the concurrent examination of affective markers with markers of cognitive decline, for the potential determination of an $\mathrm{AD}$ diagnosis. Indeed, premorbid depression has been found to act as a risk factor for the development of AD (Ownby et al. Archives of General Psychiatry 2006, 63 530-538). The significance of an integrative neuroscience approach to the assessment of AD and other forms of dementia is supported by these findings. 